\title{
Genotyping of non-human primate models: perspectives and challenges for the implementation of the "three R's"
}

\author{
T. Haus ${ }^{1}$, K. Prinz ${ }^{1}$, B. Pauling ${ }^{1}$, and C. Roos $^{2}$ \\ ${ }^{1}$ EUPRIM-Net, Research Coordination, German Primate Center, Leibniz Institute for Primate Research, \\ Göttingen, Germany \\ ${ }^{2}$ Gene Bank of Primates and Primate Genetics Laboratory, German Primate Center, \\ Leibniz Institute for Primate Research, Göttingen, Germany
}

Correspondence to: T. Haus (thaus@dpz.eu)

Received: 31 July 2014 - Revised: 2 September 2014 - Accepted: 8 September 2014 - Published: 15 October 2014

\begin{abstract}
Although non-human primates (NHPs) represent only a minor fraction of all animals used in biomedical research, there is a continuous effort to further reduce, refine and replace research with NHPs in accordance with the principles of the three R's. Most of the NHP model species are genetically highly diverse, and significant variation occurs among populations of different geographic origins, particularly in macaques. Since such differences can considerably affect the outcome of biomedical experiments, genotyping represents a promising tool to refine research approaches and to reduce the number of NHPs in biomedical research. Accordingly, the European Primate Network (EUPRIM-Net) developed an anonymous online survey to evaluate possibilities and potential hindrances for the application of genotyping in NHP research. On the one hand, our results point to the importance of genetic variation in NHPs and the need to consider the genetic background for future research approaches. On the other hand, our survey identified several hindrances and limiting factors for the application of genotyping and its incorporation in research, primate husbandry and breeding. We provide some fundamental recommendations on how to meet these challenges and how genotyping can be efficiently used to refine NHP research and to reduce the number of NHPs in biomedical research in the long term.
\end{abstract}

\section{Introduction}

Non-human primates (NHPs) are widely used as model species in several fields of biomedical research. Each year more than 100000 NHPs are globally used for scientific purposes. Major consumers are the United States, Europe and Japan (Carlsson et al., 2004; Hau and Shapiro, 2006; SCHER, 2009). Tight regulations already restrict the usage of NHPs in biomedical research and require that their application provides sufficient benefits over other animal models and that no alternative methods and techniques are available (Bontrop, 2001; SCHER, 2009; EU, 2010). Accordingly, only a minor fraction of all animals used in scientific procedures are NHPs (Roelfsema and Treue, 2014). For example, in the European Union, NHPs constitute only $0.05 \%$ of the total number of animals used in research (EC, 2013). However, due to their close genetic relationship to humans, specific disciplines still depend on NHP model species, among them research on infectious diseases, in particular human immunodeficiency virus (HIV) research, neurosciences, vaccine development, drug safety and efficacy testing, and transplantation biology (Bontrop, 2001; SCHER, 2009).

In line with the principles of the three R's ("reduction, refinement, replacement"; Russel and Burch, 1959), there is a continual effort to further reduce the number of NHPs in biomedical research, to refine procedures and experiments, and to develop appropriate in vitro methods to replace NHPs wherever possible (Guhad, 2005; Editorial, 2010). Genotyping is presently discussed to represent a promising tool to contribute to the three R's in NHP research. On the one hand it can serve to characterize the genetic background and ancestry of an individual, and on the other hand it can be used to determine genetic variants of relevant genomic regions or genes regardless of the ancestry (Bosinger et al., 2011; 
Rogers and Gibbs, 2014; Haus et al., 2014). While the latter approach is already more common in some fields of biomedical research, e.g. the typing of major histocompatibility complex (MHC) haplotypes in immunogenetics and infectious disease research (e.g. Mothé et al., 2003; Mee et al., 2009, 2010; Saito et al., 2012), genotyping of study animals to determine the genetic background and ancestry of an individual lags far behind.

Many of the NHP model species are widely distributed on the Asian or African continent, and geographic populations exhibit substantial genetic differences. This is particularly relevant for the most commonly used NHP model species, like the macaques (e.g. Lankau et al., 2014), which, after humans, have the widest distribution among primates and occur over large parts of the Asian continent (Roos and Zinner, 2014). Genetic variation among (sub)species and populations are known to cause physiological and immunological differences, and as a result individuals from different populations can produce different responses to specific biomedical experiments (Reimann et al., 2005; Luetjens and Weinbauer, 2012; Rogers et al., 2013; Seekatz et al., 2013). Accordingly, genotyping may not only help to detect and explain such differences, but can also assist in the selection of animals for specific experiments.

However, to date there are uncertainties in academia and industry regarding how to adopt the potentials of modern sequencing, and how to apply and incorporate genomic analyses and data in NHP research, breeding management and husbandry. For this purpose the European Primate Network (EUPRIM-Net) initiated an anonymous online survey to collect opinions and experiences from researchers from all fields of biomedical research. Based on these results we evaluate the current situation of genotyping as well as future perspectives and hindrances for its implementation in NHP research.

\section{Methods}

\subsection{Online survey}

We developed an anonymous online questionnaire using SoSci Survey (Leiner, 2004, www.soscisurvey.de). The survey was directed to researchers from all fields of biomedicine (e.g. neurosciences, immunology, virology, transplantation, vaccine development and drug safety/efficacy testing), breeding managers and veterinarians working with NHPs. We generated a 16-item questionnaire concerning the knowledge of geographic origin and hybrid status of NHP model species housed and used, the application of genotyping, experiences in the influence of genetic variation on research results, and expected difficulties and possibilities for the implementation of genotyping in biomedical research. We further added six questions to evaluate personal background information of participants to control for responses depending on profession/position and facility type, as well as on regional differences and model species in use. All responses are only used in aggregate and do not allow for conclusions to be drawn about individual participants or institutions represented by the participants. The survey was voluntary, strictly anonymous and we did not record names, e-mails or IP addresses. The survey was limited to a 5-week period from 25 March 2014 to 30 April 2014. The items of the complete online survey are available as a supplement.

\subsection{Participant invitation}

Although this survey was primarily developed to reach European institutes and pharmaceutical industries using NHP models in biomedical research, we also invited researchers from non-European countries to obtain as much information as possible and to reach a wider range of participants (Table 1). Because we expected that opinions may differ among people of the same institute depending on position and objectives, we did not restrict the number of participants of the same institute. We generated a password-protected link, which was included in the invitation e-mail to avoid misuse by unrequested participants. However, links were not restricted to specific IP addresses and we encouraged invitees to forward the advertisement of the questionnaire to colleagues and other people working closely with NHPs in biomedical research. We further attached an informative abstract to the invitation providing background information and explaining the intention of our project. Invitations were sent to 125 e-mail addresses, including staff from European, American, Chinese, Indian, South African and Japanese institutes, as well as to members of the association of the European Primate Veterinarians (EPV).

\subsection{Data analysis}

Due to the relative small number of participants, we analysed results of the survey only descriptively. All graphs were prepared with Excel 2013 (Microsoft).

\section{Results}

\subsection{Characterization of respondents}

Of the 125 invitations, 53 (42\%) candidates participated in the survey. Forty-six $(37 \%)$ respondents finalized all items of the survey and were included in the analysis. Table 1 shows some main characteristics of participants, including current types of positions and represented facilities, as well as NHP species housed and used for experiments. The majority of participants were from Europe (70\%), reflecting the focus of this survey. We did not record responses from Japan, China and India, but in two cases no information was provided on the location of the facility. Different types of facilities were represented by the respondents: academic or research institutes, breeding facilities, and pharmaceutical industry or contract research. Most of the participants were 
Table 1. Characterization of respondents and represented facilities $(N=46)$.

\begin{tabular}{|c|c|c|c|c|c|}
\hline Position of respondents* & No. & $(\%)$ & Location of facilities/institutes & No. & $(\%)$ \\
\hline Researcher & 30 & $(65 \%)$ & Europe & 32 & $(70 \%)$ \\
\hline Veterinarian & 18 & $(39 \%)$ & USA & 9 & $(20 \%)$ \\
\hline Breeding/colony manager & 6 & $(13 \%)$ & No specification & 2 & $(4 \%)$ \\
\hline Primate husbandry & 6 & $(13 \%)$ & Southeast Asia & 1 & $(2 \%)$ \\
\hline Project/product manager & 4 & $(9 \%)$ & Africa & 1 & $(2 \%)$ \\
\hline Other & 1 & $(2 \%)$ & Mauritius & 1 & $(2 \%)$ \\
\hline NHPs used for experiments* & & & NHPs housed* & & \\
\hline Macaca & 42 & $(91 \%)$ & Macaca & 44 & $(96 \%)$ \\
\hline Callithrix & 17 & $(37 \%)$ & Callithrix & 21 & $(46 \%)$ \\
\hline Chlorocebus & 6 & $(13 \%)$ & Papio & 8 & $(17 \%)$ \\
\hline Papio & 5 & $(11 \%)$ & Chlorocebus & 5 & $(11 \%)$ \\
\hline No experiments & 3 & $(7 \%)$ & Saimiri & 1 & $(2 \%)$ \\
\hline Saimiri & 1 & $(2 \%)$ & No primates & 1 & $(2 \%)$ \\
\hline No specification & 1 & $(2 \%)$ & No specification & 1 & $(2 \%)$ \\
\hline Facility size (no. of housed primates) & & & Facility type* & & \\
\hline$>100$ individuals & 30 & $(65 \%)$ & Academic/research institute & 32 & $(70 \%)$ \\
\hline$\leq 100$ individuals & 11 & $(24 \%)$ & Breeding facility & 12 & $(26 \%)$ \\
\hline No specification & 3 & $(7 \%)$ & Pharmaceutical industry/CRO & 10 & $(22 \%)$ \\
\hline 0 & 2 & $(4 \%)$ & Other & 2 & $(4 \%)$ \\
\hline
\end{tabular}

* multiple responses possible; CRO: contract research organization

researchers $(65 \%)$ at academic or research institutes. More than $90 \%$ of represented facilities and respondents housed or used macaques (genus Macaca). The second most commonly used NHP was the common marmoset (Callithrix jacchus), whereas other primates like baboons (Papio spp.) and African green monkeys (Chlorocebus spp.) were less frequently kept or used (Table 1).

\subsection{Consideration of geographic origin, hybrid status and genetic variation}

Most participants indicated that geographic origins are well known for the NHPs housed at represented facilities (37; $80 \%)$ as well as for those NHPs which were used in experiments $(40 ; 87 \%$; Fig. 1a). We obtained similar results for the knowledge of the presence or usage of hybrid individuals at represented facilities. Eighty percent of respondents specified that hybrids were either housed $(12 ; 26 \%)$ or not housed $(25 ; 54 \%)$ at their facility. Similarly, $78 \%$ of participants reported that hybrids were used $(8 ; 17 \%)$ or not used $(28 ; 61 \%)$ for experiments at their facility. Only eight $(17 \%)$ candidates indicated uncertainty in the existence and usage of hybrids by responding with "I do not know" or "not aware of" hybrids (Fig. 1b). Furthermore, 24 (52\%) of the represented facilities put effort into maintaining pure breeding lineages from specific geographic regions or populations by selective breeding and purchasing, $14(30 \%)$ did not, and $5(11 \%)$ did not consider the geographic origin but did consider the respective species when breeding or purchasing
( $4 \%$ chose "I do not know" and $2 \%$ "we do not house primates"). Similarly, $28(61 \%)$ of respondents preferred animals from specific geographic regions or populations for their experiments. The remaining participants did not prefer populations from specific geographic regions $(8 ; 17 \%)$, or considered only the species level $(8 ; 17 \%)$; two $(4 \%)$ participants indicated that they did not conduct experiments.

We further asked participants whether they had already experienced an influence of genetic variation in NHP model species on their experimental approaches or results. Twentyone $(46 \%)$ of the respondents had already experienced an influence of genetic variation, most of whom were working in the field of infectious disease (Fig. 2). Other respondents mentioned that genetic variation could produce certain background influences (e.g. differences in stress levels), and plays a role in metabolic or autoimmune diseases and in the development of collagen-induced arthritis (CIA; "others" in Fig. 2). Only five (11\%) participants had never experienced an influence of genetic variation, and seven $(15 \%)$ respondents answered that they had not specifically tested such an influence before. The remaining contributors $(13 ; 28 \%)$ chose the option "we do not conduct experiments with primates".

\subsection{Genotyping behaviour of respondents and at represented facilities}

According to the responses, 22 (48\%) of represented facilities did not genotype primate individuals housed at their 
A

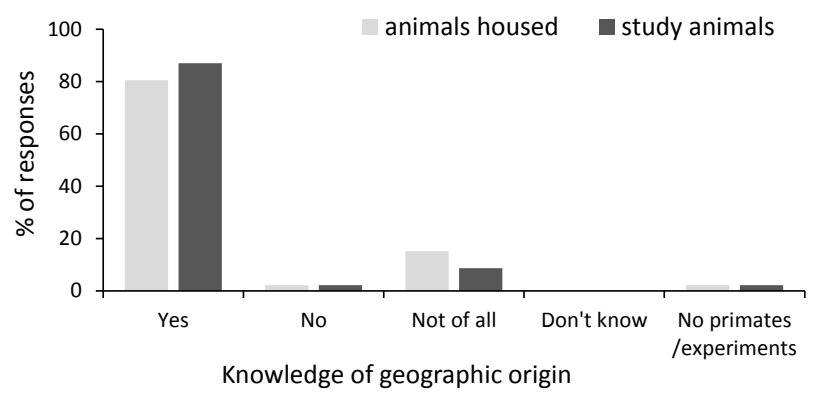

B

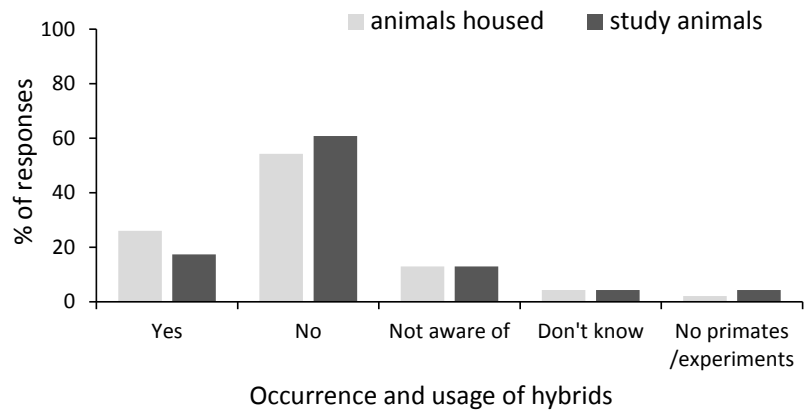

Figure 1. Knowledge of (a) geographic origin of primates housed and used in experiments, and (b) occurrence and usage of hybrids at represented facilities $(N=46)$.

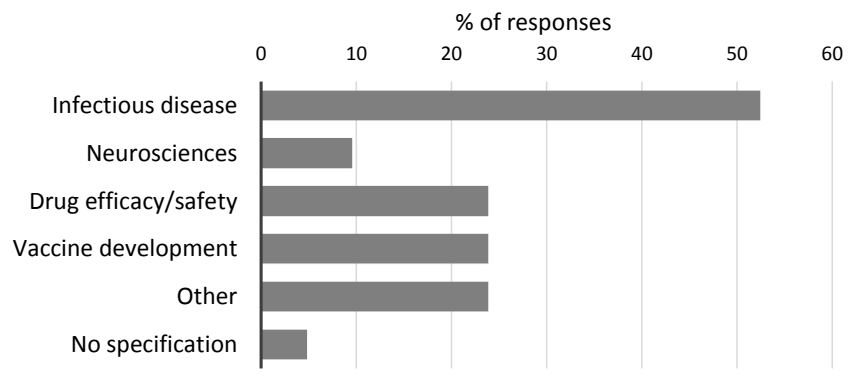

Figure 2. Frequency of experienced influence of genetic variation in major biomedical disciplines $(N=21)$.

facility. In contrast, $10(22 \%)$ participants reported that all their NHPs were genotyped, and $11(24 \%)$ stated that only individuals of specific genera or species were genotyped (Fig. 3a). Of these latter respondents, all indicated that they used to type macaque individuals (M. mulatta (22\%), M. fascicularis $(9 \%)$, M. nemestrina (2\%), and only a few participants used to genotype individuals of the genera Papio (2\%), Chlorocebus (2\%), and Callithrix (2\%). We had to exclude the response of one candidate for this item, since he/she wrongly chose response options from more than one category (see Supplement, item 7).
A

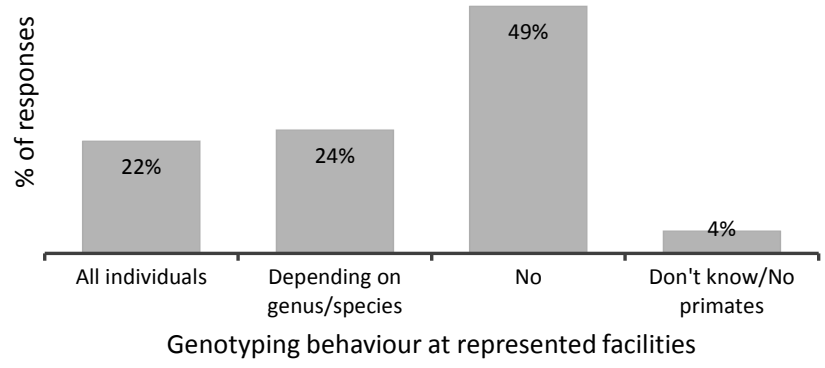

B

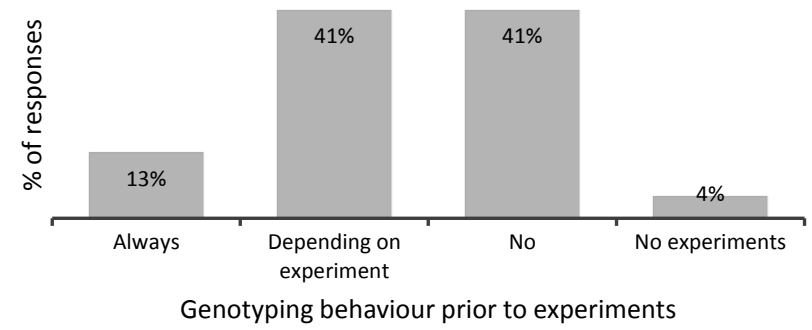

Figure 3. Genotyping behaviour (a) at represented facilities $(N=$ $45)$ and (b) before experiments $(N=46)$.

We further asked for the frequency of genotyping before animals are used in experiments. Here, 19 (41\%) respondents did not use to genotype animals before experiments, while $6(13 \%)$ of the participants indicated that study animals were always genotyped. Interestingly, 19 (41\%) respondents answered that they genotyped study animals depending on the type of experiment (Fig. 3b). Most of these participants are working in the fields of infectious disease and vaccine development (Fig. 4).

Objectives of most of the participants and facilities that conducted genotyping were the determination of geno- and haplotypes (Table 2). Only a few respondents genotyped animals to determine subspecies or geographic origins or to test for hybrids (Table 2). We further asked participants which genotyping methods and markers they would prefer in the case of genotyping. Of the 46 respondents, $39 \%$ would genotype primates at the $\mathrm{MH}$ region, while $30 \%$ would prefer single-nucleotide polymorphisms (SNPs, Fig. 5). Wholegenome and exome sequencing were chosen by only 17 and $7 \%$ of the respondents, respectively. Few others commented that methods and markers should be selected depending on the goal of the respective project. Nearly $33 \%$ of participants had no opinion regarding this question (Fig. 5). 
Table 2. Objectives for conducting genotyping at represented facilities in general ("at facility") and prior to experiments ("before experiments"). $N$ corresponds only to the number of respondents that conducted genotyping.

\begin{tabular}{lrrr}
\hline & $\begin{array}{r}\text { Detection } \\
\text { of hybrids }\end{array}$ & $\begin{array}{r}\text { (Sub)species/ } \\
\text { geographic origin }\end{array}$ & $\begin{array}{r}\text { Determination of } \\
\text { haplo/genotype }\end{array}$ \\
\hline At facility $(n=21)$ & $8(38 \%)$ & $10(48 \%)$ & $20(95 \%)$ \\
Before experiment $(n=25)$ & $7(28 \%)$ & $11(44 \%)$ & $22(88 \%)$ \\
\hline
\end{tabular}

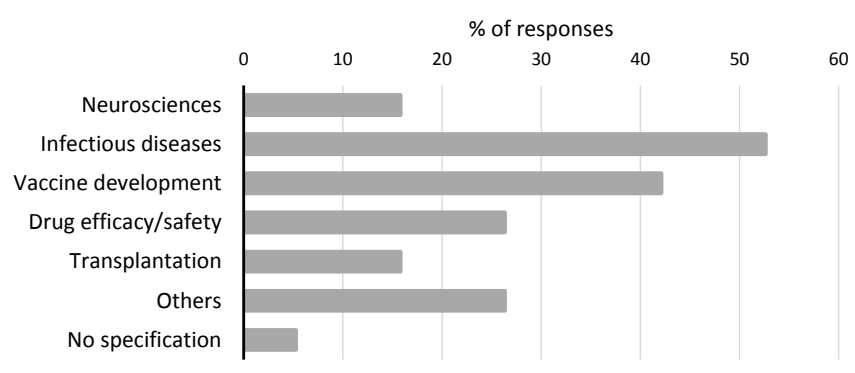

Figure 4. Genotyping frequency prior to experiments with regard to discipline $(N=19)$.

\subsection{Possibilities and hindrances for the implementation of genotyping}

Of the 46 respondents, 24 (52\%) completely agreed with the statement that genotyping prior to experiments would reduce the number of test animals and at the same time increase the quality of the results. Six (13\%) did not agree with this statement and further six $(13 \%)$ had no opinion. The remaining participants $(10 ; 22 \%)$ provided different comments; for example, they argued that genotyping might increase the quality of the results, but not that it could be used to reduce the number of test animals $(3 ; 7 \%)$, and others quoted that this supposition could not be generalized and depended on the experiment $(4 ; 9 \%)$. One participant commented that the number of test animals could not be reduced, since the number of animals in NHP studies is already very low and in some cases even statistically inadequate.

Most of the 46 participants stated that there were several hindrances or limiting factors that might impede genotyping of breeding and study animals $(28 ; 61 \%)$. Among them "limited budget" represented the most frequent factor (25; $54 \%)$. Other relevant hindrances were "limited staff" (12; $26 \%)$ and "limited time" $(11 ; 24 \%)$, and 7 (15\%) respondents indicated that the cost-benefit ratio would be too high. Other factors mentioned by the participants were the possibility of wrong genotyping results and the lack of interest of researchers. Two of them provided only a comment. Of the remaining respondents, $10(22 \%)$ did not believe that there were factors that might impede the application of genotyping of NHP model species, and $6(13 \%)$ chose the option "I do not know".

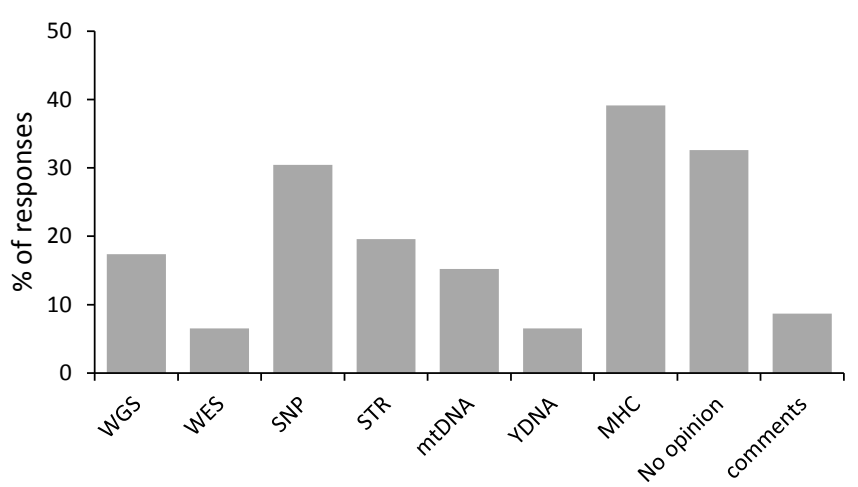

Methods and markers

Figure 5. Preferred genotyping methods and markers according to responses $(N=46)$. WGS: whole-genome sequencing; WES: whole-exome sequencing; SNP: single-nucleotide polymorphisms; STR: short tandem repeats; mtDNA: mitochondrial DNA; YDNA: Y chromosomal DNA; MHC: major histocompatibility complex.

Of the 46 respondents, 27 (59\%) indicated that there were factors which further impede the implementation of genotyping results in research approaches, primate breeding or husbandry. Among them the most frequent factor was the limited number of available NHP individuals with specific genotypes $(20 ; 44 \%)$. Other factors were difficulties in selective breeding $(16 ; 35 \%)$, e.g. artificial insemination or group housing and the maintenance of genetic diversity in breeding colonies $(14 ; 30 \%)$. Furthermore, respondents mentioned the difficulty of interpreting and analysing genotyping results as well as the lack of interest of investigators or institutes. Another fact pointed out was the limited number of vendors to order NHPs due to banning of NHP transport by airlines. Two of the respondents provided only a comment. Eleven (24\%) participants did not think that there were problems with the implementation of genotyping results, and seven $(15 \%)$ respondents chose the option "I do not know".

\section{Discussion}

Our online survey was completed by $37 \%$ of people and institutions invited, mainly from Europe and the United States. A similarly low response rate was observed in a recent survey on the use and import of NHPs for research in North America, which was completed by only $22 \%$ of invitees (Lankau 
et al., 2014). Since this survey was strictly anonymous, we were not able to control for the number of participants per institute. Accordingly, background data on represented facilities, as well as on NHP species housed or used, must be interpreted carefully. Similarly, biomedical disciplines are not equally represented by participants and do not allow for analysis of differences between disciplines. However, the high frequency of macaques in NHP research is in concordance with previous statements and studies (Carlsson et al., 2004; Fang et al., 2011; Lankau et al., 2014). We observed some discordance across response behaviours. The question "Have you ever experienced an influence of genetic variation in non-human primates on your experimental approaches or results?" (item 12, Supplement) was most probably not reliably answered by several of the 13 participants who chose "I do not conduct experiments with primates". According to personal background data (Table 1), only three respondents indicated that they did not conduct experiments with NHPs, which is also in concordance with responses to most of the other questions. We assume that this misrepresentation occurred due to the lack of "no specification" or "I do not know" options in that item. However, for most questions these options were available to avoid false-positive or negative responses (Supplement).

\subsection{Knowledge and significance of geographic origin, hybrid status and genetic variation}

Our survey identified that more than three-quarters of both the participants and the represented facilities claim to have good genetic background knowledge of their breeding and study animals with respect to geographic origin and hybrid status. Moreover, more than half of the represented facilities have an interest in the maintenance of pure breeding lineages from specific geographic regions or populations by selective breeding or purchasing, which implies a good knowledge of genetic background data of animals. The availability of pure breeding lineages from specific geographic regions is particularly important for disciplines like infectious diseases, in which the genetic make-up of study animals can have a significant influence on experimental results (e.g. Reimann et al., 2005). This is supported by $46 \%$ of the participants who had already experienced an influence of genetic variation on their results. More than half of these respondents were working in the field of infectious disease. Although less represented in this study, genetic variation also plays an influential role in neurosciences, drug and vaccine development, arthritis research, and research into metabolic or autoimmune diseases (Fig. 2).

Interestingly, $48 \%$ of represented facilities did not genotype their NHPs, and $41 \%$ of respondents did not genotype their study animals prior to experiments. Compared to the much greater number of respondents and represented facilities that specified to know the geographic origins and hybrid status of their animals $(\geq 80 \%)$, these statements must be treated carefully. It is assumed that geographic origins of imported NHPs cannot be reliably traced back, because a significant number of traded animals, especially macaques, are not directly imported from their native country of origin (Street et al., 2007; Hsu, 2011). Moreover, past hybridization events as well as different geographic origins are often not detectable based on phenotypical characters (Kanthaswamy et al., 2008, 2014; Haus et al., 2013a, b; Roos and Zinner, 2014), challenging a later species determination. Accordingly, genotyping represents the only method to reliably identify species status, geographic origin, and potential admixture with individuals from other populations or with foreign species (Yan et al., 2011; Kanthaswamy et al., 2014). Thus, with respect to the high intra- and interspecies genetic diversity as well as the not-infrequent presence of introgressed individuals in some NHP model species, our survey results identified a huge lack of genetic background knowledge in study and breeding animals. Moreover, most of the participants who conducted genotyping intended to determine geno- and haplotypes (>85\%), and only less than 50 and $30 \%$ intended to characterize the geographic origin and hybrid status, respectively (Table 2 ). We recommend that primate facilities and researchers start to pay much greater attention to the genetic make-up of their animals and to genotype their NHPs more frequently to characterize their genetic background and ancestry. This is of particular importance since macaques, which are genetically highly diverse (Street et al., 2007; Yuan et al., 2012; Kanthaswamy et al., 2014; Roos and Zinner, 2014), represent the most frequently used and housed primate genus (results of this survey; Lankau et al., 2014).

\subsection{Implementation of genotyping in research and breeding management}

Genotyping of breeding and study animals is still uncommon in biomedical research (Haus et al., 2014), which is also reflected by the results of our survey. More than half of the respondents, including those who already conduct genotyping, believed that there are different factors that hinder the application of genotyping as well as the implementation of respective results in NHP research, breeding or husbandry. Although costs for genotyping are becoming increasingly cheaper (Rogers, 2013), results of our survey indicate that the budget is still the major limiting factor for conducting genotyping at most of the represented facilities. Other problems are limited time and staff, which are usually linked to the limited budget. Preferred methods for genotyping were the analysis of MHC haplotypes and SNPs, whereas genomewide approaches like whole-genome or exome sequencing were preferred by only a few participants. While it is true that not all facilities have the capability to analyse complete genomic information of hundreds of individuals, the analysis of genome-wide information of all NHP model species on a population genetic scale represents a fundamental part 
of the progress in biomedical research (Bosinger et al., 2011; Yan et al., 2011; Rogers, 2013; Rabbani et al., 2014; Haus et al., 2014). Such an approach would substantially increase our understanding of gene variants and respective susceptibilities to diseases and disorders (Bosinger et al., 2011; Ebeling et al., 2011; Rogers and Gibbs, 2014).

More than $30 \%$ of participants had no opinion about the methods and markers of choice for genotyping. This uncertainty also includes the interpretation and analysis of genome-wide collected data, which was mentioned by some participants as hindrance for the implementation of genotyping results in biomedical research. A general problem for the implementation of genotyping might be that the fast progress in the development of modern high-throughput sequencing techniques represents a challenge for non-experts on this field. However, with a continuing development towards genetic-based research in human biomedicine, e.g. in personalized medicine (Simon and Roychowdhury, 2013; Workman et al., 2013), primate facilities and institutes working with NHPs need to invest in specialized staff to manage these challenges.

Another important factor is the limited number of available individuals of a specific genotype, which was the most frequently mentioned hindrance in this survey. If genotyping becomes a common tool in biomedical research, requests for individuals of specific genotypes will inevitably increase. One possibility to accommodate such a demand is to selectively breed eligible genotypes for specific research fields. However, this will be a long process due to the slow reproduction of NHPs (Carlsson et al., 2004). European and US breeding colonies are not able to supply the current demand for NHPs for biomedical research, and researchers and industries still rely on the import of thousands of NHPs from Southeast Asia, China, Mauritius and Israel yearly (Goodman and Check, 2002; Carlsson et al., 2004; Lankau et al., 2014). Moreover, despite the request for specific genotypes today, we have to ensure that the genetic diversity of NHP models in captivity is maintained for future research fields, restricting the extent of selective breeding (results of this study; Haus et al., 2014). Other problems derive from primate husbandry. Artificial insemination is non-trivial in many NHP species and not consistent with other efforts within the framework of the three R's like group housing and the general refinement of procedures.

Despite these hindrances, $59 \%$ of participants expected that the usage of genotyped individuals would increase the reliability and efficacy of the results, and $52 \%$ of the respondents believed that the number of NHPs in experiments could be reduced through genotyping. As a first step, we recommend that much more emphasis be put into separating and maintaining pure breeding lineages from specific geographic regions and populations as outlined in Haus et al. (2014). Subsequently, the establishment of genomic data banks for these colonies would highly advance NHP research by enabling a better exchange of information, NHPs and NHP material of specific haplotypes across facilities - something that has already been started up by the National Primate Research Centers (NPRCs) in the US (Ferguson et al., 2009; Kanthaswamy et al., 2009). Such approaches can further reduce the background noise in biomedical experiments, since they allow for selection of study subjects from the same geographic origins for a single experiment (Haus et al., 2014). This might be particularly beneficial with regard to the occurrence of introgression as a result of past hybridization in macaques and other NHP model species, because individuals from the same geographic origins are usually equally affected by genomic admixture (Stevison and Kohn, 2009; Roos and Zinner, 2014).

\section{Conclusions}

Genetic variation of NHP model species can affect the outcome of experiments in diverse biomedical disciplines. Accordingly, there is an increasing trend of considering the geographic origins of study subjects, which can be reliably determined only on a genetic basis. Our survey results indicate that genotyping to characterize the genetic background of study animals needs much more consideration in future biomedical research. To this end, genotyping has a great potential to reduce the number of animals in NHP research and to refine experimental approaches and the reliability of results. Thereby comparative whole-genome sequencing data might represent an essential basis for the application of genotyping in future research. Once genomic data sets are established, a wide range of researchers should be able to use markers like MHC and SNPs, which are the most promising tools to genotype and select study subjects depending on the experiment.

Author contributions. T. Haus, K. Prinz, B. Pauling and C. Roos designed the project and developed the online survey. T. Haus conducted the analysis and wrote the manuscript with contributions from all co-authors.

\section{The Supplement related to this article is available online at doi:10.5194/pb-1-1-2014-supplement.}

Acknowledgements. We thank Robert Teepe, Gerhard Weinbauer and Uwe Schönmann for providing helpful discussions and comments during the development of the online survey, as well as Stefan Treue for commenting on a previous version of the manuscript. We are grateful to all respondents for participating in our survey. This work has been supported by EUPRIM-Net under the EU grant agreement 262443 of the 7th Framework Programme.

Edited by: J. Tung

Reviewed by: S. T. Shipley and one anonymous referee 


\section{References}

Bontrop, R. E.: Non-human primates: essential partners in biomedical research, Immunological Rev., 183, 5-9, doi:10.1034/j.1600065x.2001.1830101.x, 2001.

Bosinger, S. E., Johnson, Z. P., and Silvestri, G.: Primate genomes for biomedicine, Nat. Biotechnol., 29, 983-4, doi:10.1038/nbt.2032, 2011.

Carlsson, H.-E., Schapiro, S. J., Farah, I., and Hau, J.: Use of primates in research: a global overview, Am. J. Primatol., 63, 225237, 2004.

Ebeling, M., Küng, E., See, A., Broger, C., Steiner, G., Berrera, M., Heckel, T., Iniguez, L., Albert, T., Schmucki, R., Biller, H., Singer, T., and Certa, U.: Genome-based analysis of the nonhuman primate Macaca fascicularis as a model for drug safety assessment, Genome Res., 21, 1746-1756, doi:10.1101/gr.123117.111, 2011.

EC (European Commission): Seventh Report on the Statistics on the Number of Animals used for Experimental and other Scientific Purposes in the Member States of the European Union, available at: http://eur-lex.europa.eu/resource.html?uri=cellar: d2e73ac5-60d0-11e3-ab0f-01aa75ed71a1.0001.01/DOC_ 1\&format=_PDF (last access: 13 June 2014), 2013.

Editorial: Reduce, refine, replace, Nat. Immunol., 11, 971, doi:10.1038/ni1110-971, 2010.

EU (European Union): Directive 2010/63/EU of the European Parliament and the Council on the protection of animals used for scientific purposes, Official Journal of the European Union, L276/33, available at: http://eur-lex.europa.eu/LexUriServ/LexUriServ.do?uri=OJ: L:2010:276:0033:0079:en:PDF (last access: 19 May 2014), 2010.

Fang, X., Zhang, Y., Zhang, R., Yang, L., Li, M., Ye, K., Guo, X., Wang, J. and Su, B. Genome sequence and global sequence variation map with 5.5 million SNPs in Chinese rhesus macaque, Genome. Biol., 12, R63, doi:10.1186/gb-2011-12-7-r63, 2011.

Ferguson, B., Capitanio, J., Folks, T., Hotchkiss, C., Johnson, Z., Kean, L., Kubisch, H. M., Lank, S., Lyons, L., Miller, G. M., Nylander, J., O'Connor, D., Vallender, E. J., and Wiseman, R.: Resource brief: the National Non-Human Primate DNA Bank, Methods, 49, 3-4, doi:10.1016/j.ymeth.2009.07.011, 2009.

Goodman, S. and Check, E.: The great primate debate, Nature, 417 , 684-687, doi:10.1038/417684a, 2002.

Guhad, F.: Introduction to the 3Rs (refinement, reduction and replacement), Contemp. Top. Lab. Anim. Sci., 44, 58-59, 2005.

Hau, J. and Schapiro, S. J.: Non-human primates in biomedical research, Scand. J. Lab. Anim. Sci., 33, 9-12, 2006.

Haus, T., Akom, E., Agwanda, B., Hofreiter, M., Roos, C., and Zinner, D.: Mitochondrial diversity and distribution of African green monkeys (Chlorocebus Gray, 1870), Am. J. Primatol., 75, 350360, doi:10.1002/ajp.22113, 2013a.

Haus, T., Roos, C., and Zinner, D.: Discordance Between Spatial Distributions of Y-Chromosomal and mitochondrial haplotypes in African green monkeys (Chlorocebus spp.): a result of introgressive hybridization or cryptic diversity?, Int. J. Primatol., 34, 986-999, doi:10.1007/s10764-013-9717-5, 2013b.

Haus, T., Ferguson, B., Rogers, J., Doxiadis, G., Certa, U., Rose, N. J., Teepe, R., Weinbauer, G. F., and Roos, C.: Genome typing of nonhuman primate models: implications for biomedical research,
Trends Genet., 1-6, doi:10.1016/j.tig.2014.05.004, online first, 2014.

Hsu, C. K.: China as a resource for NHP, in: Animal Research in a Global Environment - Meeting the Challenges, 224-227, The National Academies Press, Washington D.C., 2011.

Kanthaswamy, S., Satkoski, J., George, D., Kou, A., Erickson, B. J.-A., and Smith, D. G.: Interspecies hybridization and the stratification of nuclear genetic variation of rhesus (Macaca mulatta) and long-tailed macaques (Macaca fascicularis), Int. J. Primatol., 29, 1295-1311, doi:10.1007/s10764-008-9295-0, 2008.

Kanthaswamy, S., Capitanio, J. P., Dubay, C. J., Ferguson, B., Folks, T., Ha, J. C., Hotchkiss, C. E., Johnson, Z. P., Katze, M. G., Kean, L. S., Kubisch, H. M., Lank, S., Lyons, L. a, Miller, G. M., Nylander, J., O'Connor, D. H., Palermo, R. E., Smith, D. G., Vallender, E. J., Wiseman, R. W., and Rogers, J.: Resources for genetic management and genomics research on non-human primates at the National Primate Research Centers (NPRCs), J. Med. Primatol., 38, 17-23, doi:10.1111/j.1600-0684.2009.00371.x, 2009.

Kanthaswamy, S., Johnson, Z., Trask, J. S., Ramakrishnan, R., Bahk, J., Ng, J., Wiseman, R., Kubisch, H. M., Vallender, E. J., Smith, D. G., Rogers, J., and Ferguson, B.: Development and validation of a SNP-based assay for inferring the genetic ancestry of rhesus macaques (Macaca mulatta), Am. J. Primatol., doi:10.1002/ajp.22290, online first, 2014.

Lankau, E. W., Turner, P. V., Mullan, R. J., and Galland, G. G.: Use of nonhuman primates in research in North America, J. Am. Assoc. Lab. Anim. Sci., 53, 278-282, 2014.

Leiner, D. J.: SoSci Survey (Version 2.4.00-i), Computer Software, available at: https://www.soscisurvey.de, last access: 30 April 2014.

Luetjens, C. M. and Weinbauer, G. F.: Functional assessment of sexual maturity in male macaques (Macaca fascicularis), Regul. Toxicol. Pharmacol., 63, 391-400, 2012.

Mee, E. T., Berry, N., Ham, C., Sauermann, U., Maggiorella, M. T., Martinon, F., Verschoor, E. J., Heeney, J. L., Le Grand, R., Titti, F., Almond, N., and Rose, N. J.: Mhc haplotype H6 is associated with sustained control of SIVmac251 infection in Mauritian cynomolgus macaques, Immunogenetics, 61, 327-39, doi:10.1007/s00251-009-0369-8, 2009.

Mee, E. T., Berry, N., Ham, C., Aubertin, a, Lines, J., Hall, J., Stebbings, R., Page, M., Almond, N., and Rose, N. J.: Mhc haplotype M3 is associated with early control of SHIVsbg infection in Mauritian cynomolgus macaques, Tissue Antigens, 76, 223-229, doi:10.1111/j.1399-0039.2010.01500.x, 2010.

Mothé, B. R., Weinfurter, J., Wang, C., Rehrauer, W., Wilson, N., Allen, T. M., Allison, D. B., and Watkins, D. I.: Expression of the Major Histocompatibility Complex Class I Molecule Mamu-A * 01 is associated with control of simian immunodeficiency virus SIV mac 239 replication, J. Virol., 77, 2736, doi:10.1128/JVI.77.4.2736, 2003.

Rabbani, B., Tekin, M., and Mahdieh, N.: The promise of wholeexome sequencing in medical genetics, J. Hum. Genet., 59, 5-15, doi:10.1038/jhg.2013.114, 2014.

Reimann, K. A., Parker, R. A., Seaman, M. S., Beaudry, K., Beddall, M., Peterson, L., Williams, K. C., Veazey, R. S., Montefiori, D. C., Mascola, J. R., Nabel, G. J., and Letvin, N. L.: Pathogenicity of simian-human immunodeficiency virus SHIV-89.6P and SIVmac is attenuated in cynomolgus macaques and associated 
with early T-lymphocyte responses, J. Virol., 79, 8878-8885, doi:10.1128/JVI.79.14.8878, 2005.

Roelfsema, P. R. and Treue, S.: Basic neuroscience research with nonhuman primates: a small but indispensable component of biomedical research, Neuron, 82, 1200-1204, 2014.

Rogers, J.: In transition: primate genomics at a time of rapid change, ILAR J., 54, 224-233, 2013.

Rogers, J. and Gibbs, R. A: Comparative primate genomics: emerging patterns of genome content and dynamics, Nat. Rev. Genet., 15, 347-359, doi:10.1038/nrg3707, 2014.

Rogers, J., Raveendran, M., Fawcett, G. L., Fox, a S., Shelton, S. E., Oler, J. A, Cheverud, J., Muzny, D. M., Gibbs, R. A, Davidson, R. J., and Kalin, N. H.: CRHR1 genotypes, neural circuits and the diathesis for anxiety and depression, Mol. Psychiatry, 18, 700707, doi:10.1038/mp.2012.152, 2013.

Roos, C. and Zinner, D: Diversity and evolutionary history of macaques with special focus on rhesus and long-tailed macaques, in: The Nonhuman Primate in Nonclinical Drug Development and Safety Assessment, edited by: Blümel, J., Korte, S., Schenck, E., and Weinbauer, G. F., Elsevier, in press, 2014.

Russell, W. M. S. and Burch, R. L.: The principles of humane experimental technique, Methuen and Co. Ltd, London, 1959.

Saito, Y., Naruse, T. K., Akari, H., Matano, T., and Kimura, A.: Diversity of MHC class I haplotypes in cynomolgus macaques, Immunogenetics, 64, 131-141, doi:10.1007/s00251-011-0568-y, 2012.

SCHER (Scientific Committee on Health and Environmental Risks): The need for non-human primates in biomedical research, production and testing of products and devices, European Commission, 38 pp., available at: http://ec.europa.eu/health/ph_ risk/committees/04_scher/docs/scher_o_110.pdf (last access: 27 June 2014), 2009.

Seekatz, A. M., Panda, A., Rasko, D. A., Toapanta, F. R., EloeFadrosh, E. A, Khan, A. Q., Liu, Z., Shipley, S. T., Detolla, L. J., Sztein, M. B., and Fraser, C. M.: Differential response of the cynomolgus macaque gut microbiota to Shigella infection, PLoS One, 8, e64212, doi:10.1371/journal.pone.0064212, 2013.
Simon, R. and Roychowdhury, S.: Implementing personalized cancer genomics in clinical trials, Nat. Rev. Drug Discov. 12, 358369, 2013.

Stevison, L. S. and Kohn, M. H.: Divergence population genetic analysis of hybridization between rhesus and cynomolgus macaques, Mol. Ecol., 18, 2457-2475, doi:10.1111/j.1365294X.2009.04212.x, 2009.

Street, S. L., Kyes, R. C., Grant, R., and Ferguson, B.: Single nucleotide polymorphisms (SNPs) are highly conserved in rhesus (Macaca mulatta) and cynomolgus (Macaca fascicularis) macaques, BMC Genomics, 8, 480, doi:10.1186/1471-2164-8480, 2007.

Workman, P., Al-Lazikani, B., and Clarke, P. A.: Genome-based cancer therapeutics: targets, kinase drug resistance and future strategies, Curr. Opin. Pharmacol., 13, 486-496, 2013.

Yan, G., Zhang, G., Fang, X., Zhang, Y., Li, C., Ling, F., Cooper, D. N., Li, Q., Li, Y., Gool, A. J. Van, Du, H., Chen, J., Chen, R. Zhang, P., Huang, Z., Thompson, J. R., Meng, Y., Bai, Y., Wang, J., Zhuo, M., Wang, T., Huang, Y., Wei, L., Li, J., Wang, Z., Hu, H., Yang, P., Le, L., Stenson, P. D., Li, B., Liu, X., Ball, E. V, An, N., Huang, Q., Zhang, Y., Fan, W., Zhang, X., Li, Y., Wang, W., Katze, M. G., Su, B., Nielsen, R., and Yang, H.: Genome sequencing and comparison of two nonhuman primate animal models, the cynomolgus and Chinese rhesus macaques, Nat. Biotechnol., 29, 1019-1025, doi:10.1038/nbt.1992, 2011.

Yuan, Q., Zhou, Z., Lindell, S. G., Higley, J. D., Ferguson, B., Thompson, R. C., Lopez, J. F., Suomi, S. J., Baghal, B., Baker, M., Mash, D. C., Barr, C. S., and Goldman, D.: The rhesus macaque is three times as diverse but more closely equivalent in damaging coding variation as compared to the human, BMC Genet., 13, 52, doi:10.1186/1471-2156-13-52, 2012. 